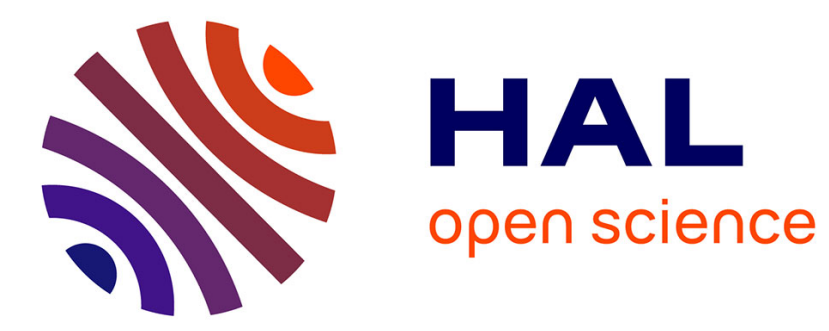

\title{
Force-field adaptation without proprioception: Can vision be used to model limb dynamics?
}

Fabrice R. Sarlegna, Nicole Malfait, Lionel Bringoux, Christophe Bourdin, Jean-Louis Vercher

\section{- To cite this version:}

Fabrice R. Sarlegna, Nicole Malfait, Lionel Bringoux, Christophe Bourdin, Jean-Louis Vercher. Forcefield adaptation without proprioception: Can vision be used to model limb dynamics?. Neuropsychologia, 2010, 48 (1), pp.60-67. 10.1016/j.neuropsychologia.2009.08.011 . hal-01384815

\section{HAL Id: hal-01384815 https://hal.science/hal-01384815}

Submitted on 4 May 2018

HAL is a multi-disciplinary open access archive for the deposit and dissemination of scientific research documents, whether they are published or not. The documents may come from teaching and research institutions in France or abroad, or from public or private research centers.
L'archive ouverte pluridisciplinaire HAL, est destinée au dépôt et à la diffusion de documents scientifiques de niveau recherche, publiés ou non, émanant des établissements d'enseignement et de recherche français ou étrangers, des laboratoires publics ou privés. 


\title{
Force-field adaptation without proprioception: Can vision be used to model limb dynamics?
}

\author{
Fabrice R. Sarlegna ${ }^{\mathrm{a}, \mathrm{b}, *}$, Nicole Malfait ${ }^{\mathrm{c}}$, Lionel Bringoux ${ }^{\mathrm{a}}$, Christophe Bourdin $^{\mathrm{a}}$, \\ Jean-Louis Vercher ${ }^{\mathrm{a}}$ \\ a Institut des Sciences du Mouvement, CNRS E' Université de la Méditerranée, 163 Avenue de Luminy, 13288 Marseille, France \\ b Laboratoire de Neurobiologie de la Cognition, CNRS \& Université de Provence, 3 place Victor Hugo, 13331 Marseille, France \\ ${ }^{\mathrm{c}}$ Laboratoire de Neurosciences Intégratives et Adaptives, CNRS \& Université de Provence 3 Place Victor Hugo, 13331 Marseille, France
}

\section{A R T I C L E I N F O}

\section{Article history:}

Received 22 December 2008

Received in revised form 3 August 2009

Accepted 11 August 2009

Available online 18 August 2009

\section{Keywords:}

Motor learning

Internal representation

Goal-directed arm movements

Reaching

Proprioceptive deafferentation

Sensory substitution

\begin{abstract}
A B S T R A C T
Because our environment and our body can change from time to time, the efficiency of human motor behavior relies on the updating of the neural processes transforming intentions into actions. Adaptation to the context critically depends on sensory feedback such as vision, touch or hearing. Although proprioception is not commonly listed as one of the main senses, its role is determinant for the coordination of daily gestures like goal-directed arm movements. In particular, previous work suggests that proprioceptive information is critical to update the internal representation of limb dynamic properties. Here, we examined the motor behavior of a deafferented patient, deprived of proprioception below the nose, to assess adaptation to new dynamic conditions in the absence of limb proprioception. The patient, and age-matched control participants, reached toward visual targets in a new force field created by a rotating platform. Full vision of the limb and workspace was available throughout the experiment. Although her impairment was obvious in baseline reaching performance, the proprioceptively deafferented patient clearly adapted to the new force conditions. In fact, her time course of adaptation was similar to that observed in controls. Moreover, when tested in the normal force field after adaptation to the new force field, the patient exhibited after-effects similar to those of controls. These findings show that motor adaptation to a modified force field is possible without proprioception and that vision can compensate for the permanent loss of proprioception to update the central representation of limb dynamics.
\end{abstract}

(c) 2009 Elsevier Ltd. All rights reserved.

\section{Introduction}

An intriguing issue in behavioral neuroscience is how the brain selects and combines sensory signals to transform intentions into actions. It has been suggested that sensory signals are processed differently as a function of task requirements (Krakauer, Ghilardi, \& Ghez, 1999; Sainburg, Lateiner, Latash, \& Bagesteiro, 2003; Sober \& Sabes, 2005; for a review). In particular, the initial planning stage of goal-directed arm movements would mainly rely on visual inputs to determine the position of the target relative to the hand, whereas proprioceptive information would be more crucial to specify the final motor commands which take into account the physical properties of the musculoskeletal system Sarlegna \& Sainburg, 2009.

The critical role of proprioception in motor control has been shown in numerous experiments on healthy humans (e.g., Brown, Rosenbaum, \& Sainburg, 2003; Cordo, Gurfinkel, Bevan, \& Kerr,

* Corresponding author at: Laboratoire de Neurobiologie de la Cognition, CNRS \& Université de Provence, 3 place Victor Hugo, 13331 Marseille, France. Tel.: +33 6 663236 54; fax: +33488576872.

E-mail address: fabrice.sarlegna@gmail.com (F.R. Sarlegna).
1995 ) and is also well demonstrated by studies on proprioceptively deafferented patients. The pioneer work of Mott and Sherrington (1895) on non-human primates showed that, after deafferentation by dorsal root section, motor behavior was severely impaired (see also Gauthier \& Mussa Ivaldi, 1988; Polit \& Bizzi, 1979; Taub \& Goldberg, 1974). In humans, the rare clinical cases of proprioceptive deafferentation confirmed the crucial involvement of proprioception in movement coordination (Rothwell et al., 1982; Messier, Adamovich, Berkinblit, Tunik, \& Poizner, 2003; Sanes, Mauritz, Dalakas, \& Evarts, 1985; Sarlegna, Gauthier, Bourdin, Vercher, \& Blouin, 2006; Vercher et al., 1996). Sainburg, Ghilardi, Poizner and Ghez (1995) demonstrated that the deficits of deafferented patients to control multi-joint arm movements reflect their inability to compensate in a predictive manner for the interaction torques arising at one joint due to the motions of adjacent limb segments. Sainburg et al. (1995) suggested that proprioception was critical to update an internal model of limb dynamics used to control reaching movements.

An internal representation of limb dynamic properties is necessary because the effect of the motor commands depends on the effector and the environment. The remarkable plasticity of the 
representation of limb dynamics has been demonstrated by studying the adaptation to new force environments (Lackner \& DiZio, 1994; Shadmehr \& Mussa-Ivaldi, 1994). When arm movements are perturbed from the intended trajectory by new forces, somatosensory as well as visual error signals may drive the adaptive update of the sensori-motor transformations. However, for velocity- and position-dependent force fields, no major differences in adaptation have been reported between movements performed with or without hand visual feedback (Franklin, So, Burdet, \& Kawato, 2007; Scheidt, Conditt, Secco, \& Mussa-Ivaldi, 2005; Tong, Wolpert, \& Flanagan, 2002). Only with more complex, multiple-force environments has it been shown that visual feedback substantially contributes to motor adaptation (Bourdin, Gauthier, Blouin, \& Vercher, 2001; Bourdin, Bringoux, Gauthier, \& Vercher, 2006). In fact, it has been clearly demonstrated that for position-, velocityand acceleration-dependent force fields, somatosensory feedback is sufficient to drive dynamic adaptation (e.g., Coello, Orliaguet, \& Prablanc, 1996; Krakauer et al., 1999; Lackner \& DiZio, 1994; Shadmehr \& Mussa-Ivaldi, 1994; Tong et al., 2002). This was clearly demonstrated by DiZio and Lackner (2000) who asked participants to reach toward visual targets in complete darkness. The rotation of the experimental platform modified the force-field environment such that initial reaching movements in the novel field were significantly deviated as a function of movement velocity. Healthy participants could adapt to the new force field despite the absence of visual or tactile feedback about reaching performance as they could rapidly produce accurate movements with straight trajectories, like in the usual force field. Similar findings were obtained with congenitally blind patients, supporting the idea that the central representation of limb dynamics can be efficiently updated on the sole basis of proprioception. In a recent study, Pipereit, Bock and Vercher (2006) showed that degrading proprioceptive signals with the vibration technique substantially impaired motor adaptation to a velocity-dependent, mechanical perturbation, further suggesting that intact proprioception is critical for dynamic adaptation.

Previous research highlights the prominent role of proprioceptive inputs for the adaptive control of limb dynamics. In the present study, we asked whether proprioception is necessary to model limb dynamics by testing how a rare patient, totally deprived of limb proprioception, could adapt her reaching behavior to a novel force environment created by a rotating platform. Because deafferented patients cannot produce compensatory responses to force perturbations without vision (Nougier et al., 1996; Rothwell et al., 1982; Sanes et al., 1985), we elected to allow full vision of reaching performance to the patient as well as to the control participants. Based on previous studies (Ghez, Gordon, \& Ghilardi, 1995; Sainburg, Poizner, \& Ghez, 1993), we hypothesized that vision might partially compensate for the loss of proprioception to control arm movements in the new dynamic environment.

\section{Methods}

\subsection{Subjects}

One 60-year-old proprioceptively deafferented patient and 6 healthy, agematched control subjects participated in this experiment. Participants gave their informed consent prior to the study, in accordance with the ethical standards set out in the 1964 Declaration of Helsinki. All participants were self-declared right-handed and had normal or corrected-to-normal vision. None of the controls ( 3 females and 3 males, mean age $=57 \pm 4$ years; data present mean \pm inter-individual standard deviation of the mean throughout this manuscript) had any relevant medical history. Al participants were naive to the experiment.

The deafferented patient, known as GL, had two severe episodes (at the ages of 27 and 31 years) of extensive polyneuropathy, affecting her whole body below the nose. Clinically, she suffers from a complete loss of touch, vibration, pressure and kinesthetic senses below the neck and presents no tendon reflexes in the four limbs Cooke, Brown, Forget and Lamarre (1985) reported that (i) no sensory potentials can be recorded from her hands or feet and no cortical response can be evoked by electrical stimulation of the peripheral nerves of either arm (ii) $\mathrm{H}$ reflexes are absent in the legs (iii) a sural nerve biopsy revealed that the density of the myelinated fibers ( 2496 fibers $/ \mathrm{mm}^{2}$ ) is much lower than normal (more than 6000 fibers $/ \mathrm{mm}^{2}$ ), while the percentage of myelinated fibers larger than $9 \mu \mathrm{m}$ in diameter is very small $(0.31 \%)$ with respect to normal values (more than $18 \%$ ).

Pain and temperature can be felt, indicating a selective impairment of the large-diameter peripheral sensory myelinated fibers. The loss of inputs from both cutaneous mechanoreceptors (as shown by deficits in tactile sensitivity in Olausson et al., 2002) and muscle receptors (as shown by loss of stretch reflexes) means that GL's neuropathy is not selective for subtypes of large sensory myelinated fibers. The motor fibers are not affected as shown by motor nerve conduction velocities and needle electromyography investigation of the arm muscles (Cooke et al., 1985). Functionally, GL remains largely confined to a wheelchair but can perform daily manual tasks by using visual information and attentional resources (Blouin et al., 1993; Ghez et al., 1995; see also Ingram et al., 2000; Rothwell et al., 1982). No sign of recovery has been observed in periodical tests made since her second episode of deafferentation left her without proprioception below the nose.

\subsection{Experimental set-up}

Participants were seated at the center of a motorized platform and were asked to reach toward visual targets. A bucket seat, an adjustable headrest and a four-point belt were used to restrain the head and trunk. The limb workspace was always illuminated while an opaque structure prevented participants from viewing the walls of the experimental room. On a horizontal board, at waist level, a landmark indicated the hand starting position and the visual targets were red light-emitting diodes ( $3 \mathrm{~mm}$ in diameter). Three targets were positioned on a $37 \mathrm{~cm}$ radius circular array at $0^{\circ}$ (straight-ahead), $20^{\circ}$ (to the right) and $-20^{\circ}$ with respect to start position.

An infrared active marker was taped to the right index fingertip whose position was sampled at $500 \mathrm{~Hz}$ using an optical motion tracking system (Codamotion Cx1, Charnwood Dynamics Ltd, Leicestershire, UK). The experimenter controlled the tracking system, the motorized platform and the presentation of the visual targets from an adjacent room by using a customized software (Docometre) governing a real-time acquisition system ADwin-Pro (Jäger, Germany).

\subsection{Procedure}

At the beginning of each trial, participants had to actively position their right hand at the starting location. They were asked to reach as fast and accurately as possible toward visual targets with their right hand. No explicit instructions were given with respect to hand path. However, participants were required to reach in one continuous movement and not to correct after their finger contacted the horizontal board. They were also asked to maintain their left arm on the left thigh throughout the experiment. All participants were familiarized with the task during a preliminary phase.

The experimental session consisted of three conditions:

- PRE-rotation test: Participants executed 30 reaching movements (10 trials per target) while the platform remained stationary, providing baseline reaching performance in the normal force field.

- PER-rotation test: Participants performed 90 movements (30 trials per target) while the platform was rotating counterclockwise, generating clockwise Coriolis forces on the moving limb. Coriolis forces (Fcor) are proportional to the product of the platform's angular velocity (pv), arm mass ( $\mathrm{m}$ ) and tangential arm velocity (av) according to the equation: Fcor $=-2 \mathrm{~m}$.pv.av.

- POST-rotation test: This condition was similar to the PRE-rotation condition since participants performed 30 movements (10 trials per target) while the platform was stationary.

In each condition, targets were presented in a pseudo-random order, excepted for the straight-ahead, central target which was presented first in PER- and POSTrotation conditions. The order of target presentation was similar for all subjects. For the PER-rotation condition, the rotating platform was accelerated in $110 \mathrm{~s}$ up to a constant velocity of $120 \%$ s, i.e., $20 \mathrm{rpm}$. Participants were instructed not to move during acceleration and for $90 \mathrm{~s}$ afterward. The $90 \mathrm{~s}$ delay allowed the vestibular semicircular canals, activated during acceleration, to return to their resting discharge frequency. A similar procedure was employed for the deceleration between the PER- and the POST-rotation tests. The PER-rotation test lasted approximately $15 \mathrm{~min}$, the complete experimental session lasting approximately $45 \mathrm{~min}$.

\subsection{Data analysis}

Right index fingertip position signals were low-pass Butterworth-filtered at $10 \mathrm{~Hz}$, and numerically differentiated. Movement onset was defined as the first time tangential hand velocity reached $2 \mathrm{~cm} / \mathrm{s}$ and movement offset as the first time hand velocity dropped below $1 \mathrm{~cm} / \mathrm{s}$. We used such a low cut-off threshold to provide endpoint data reflecting both motor planning and online, corrective processes. Endpoint error was defined as the absolute distance between the target and the index fingertip at movement offset. Because the force field created a lateral hand path deviation, the lateral, signed error at movement offset was computed. We also computed the signed amplitude and time to maximum perpendicular deviation of the 
(A)

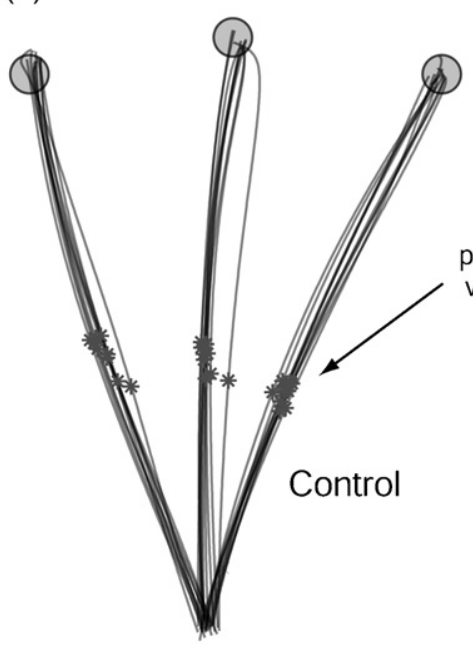

(C)

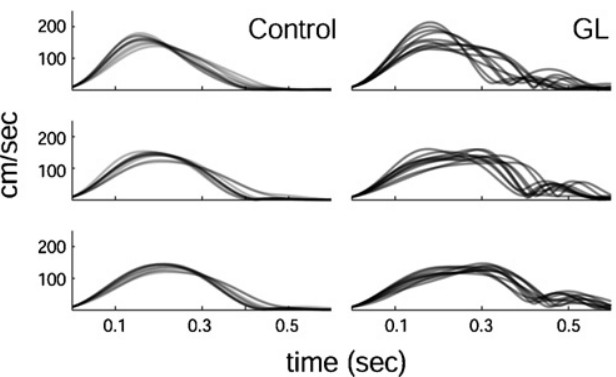

(B)
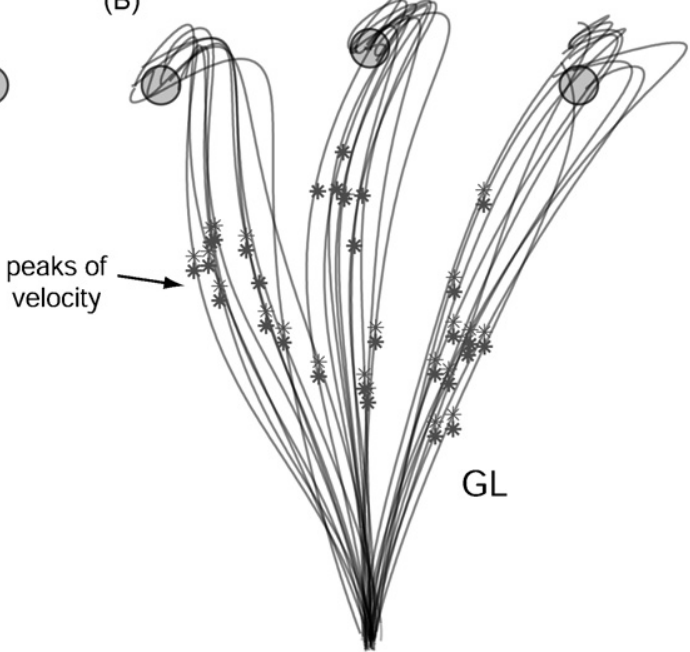

(D)

$\mathrm{GL}$

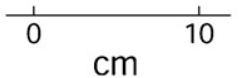

$\mathrm{cm}$

\section{Final position Initial direction Initial direction error (cm) error (deg) variability (deg)}
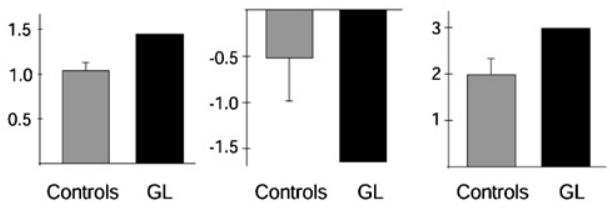

Fig. 1. Reaching behavior of a proprioceptively deafferented patient (GL) and healthy, aged-matched controls. (A and B) Top view of hand paths toward visual targets (grey circles). The representative control participant (A) and patient GL (B) had full vision of the reaching limb and workspace. Asterisks correspond to peak velocity. (C) Velocity profiles of the same control participant and patient GL for the left target (top panel), central target (middle panel) and right target (bottom panel). (D) Endpoint constant errors, trajectory constant and variable errors. Error bars represent the $95 \%$ confidence interval of control subjects.

hand path from a straight line to the target (the force field creating clockwise deviations, positive values were assigned to clockwise deviations and only positive values were considered).

The magnitude of the Coriolis forces acting on arm movements during platform rotation is maximum at peak velocity. Moreover, movement kinematics at peak velocity mainly reflect the initial motor plan, especially in seniors as their feedback control system has been shown to be slower than that of younger adults (Rossit \& Harvey, 2008; Sarlegna, 2006). We thus computed the initial movement direction as the angle between the vector from the start position to the target position and the vector from the start position to the hand position at peak velocity (Krakauer et al., 1999; Scheidt et al., 2005; Wang \& Sainburg, 2005).

To assess the adaptation process of control participants, we employed a procedure similar to the one used by Lackner and DiZio (1994). For each measure, we compared the last two trials per target of the PRE-test (thus corresponding to 6 trials in the PRE-test), the first two trials per target of the PER-rotation phase and the first two trials per target of the POST-test. Data for each kinematic measure were submitted to separate $3 \times 6$ [Test (PRE-, PER-, POST-test $) \times$ Trial $(1,2,3,4,5,6)]$ analyses of variance (ANOVA) with repeated measures. Newman-Keuls tests were used for post-hoc analysis. To compare GL's data to those of control participants, we used $t$-tests comparisons of a single value to a population sample (Nougier et al., 1996). For all tests, the significance threshold was set at 0.05 .

\section{Results}

\subsection{Baseline reaching performance in a normal force field}

Fig. 1 shows the reaching movements performed by a representative control participant and the proprioceptively deafferented patient GL in the PRE-rotation condition. Typically, reaching movements of controls were accurate with roughly straight hand paths and bell-shaped velocity profiles. In contrast, GL's motor deficits were obvious. Although the patient performed accurate reaching movements, these were executed in a more variable and rather inefficient manner with respect to control participants. Most notably, late hooks were observed: they may reflect, as discussed by Buneo, Boline, Soechting and Poppele (1995), late movement corrections or an impaired control of the deceleration phase, for example with an exaggerated vertical (downwards) movement of the finger at the end of the reach. The statistical analysis of baseline reaching performance confirmed that both trajectory control and final positioning, assessed with initial movement direction and endpoint accuracy, differed between the deafferented patient and healthy, control participants.

Fig. 1D shows bar plots of kinematic measures obtained during the PRE-rotation test to compare baseline performance of the patient and the control participants. $t$-Tests for group vs. single case values showed that control participants reached to the targets more accurately than the patient when considering endpoint error $(p<0.001)$. The analysis of standard deviation of the mean endpoint error showed that controls were less variable in endpoint positioning than GL (means $=0.4$ and $0.5 \mathrm{~cm}$, respectively; $p<0.05$ ). The greater final errors of the deafferented patient were not due to a speed-accuracy tradeoff since the peak velocity of GL's movements (mean $=1.5 \mathrm{~m} / \mathrm{s} ; \mathrm{SD}=0.2 \mathrm{~m} / \mathrm{s}$ ) did not significantly differ from that of controls ( mean $=1.6 \pm 0.1 \mathrm{~m} / \mathrm{s} ; p>0.42$ ). In fact, movement duration was greater for the patient $($ mean $=591 \mathrm{~ms}$ ) with respect to controls (mean $=440 \pm 36 \mathrm{~ms} ; p<0.001)$.

Hand trajectories were straighter for controls than for the patient (Fig. 1). Indeed, controls' initial movement direction differed from that of the patient (Fig. 1D; $p<0.01$ ) and the maximum perpendicular deviation of control participants was smaller $($ mean $=1.7 \pm 0.6 \mathrm{~cm})$ than that of the patient $($ mean $=2.9 \mathrm{~cm}$; $p<0.01$ ). We also found greater variability in the patient's hand 
(A)

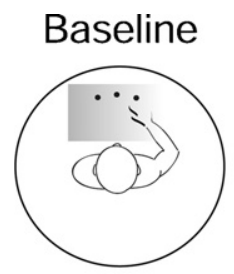

(B) Adaptation
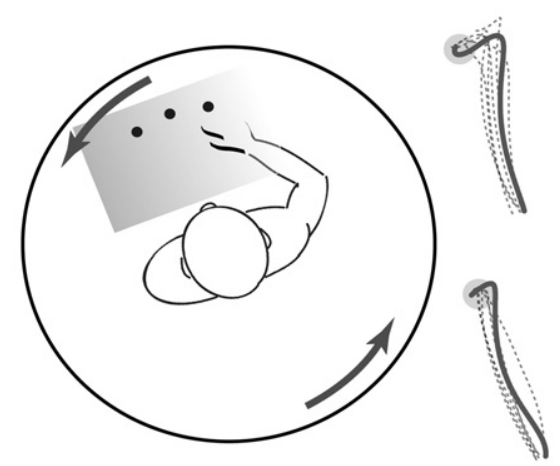

(C)
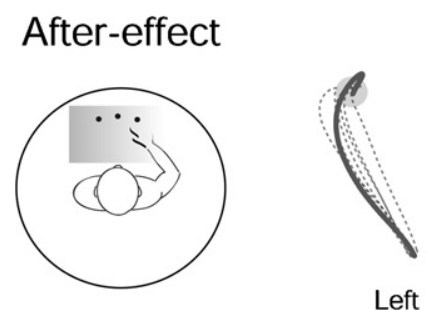

first trial
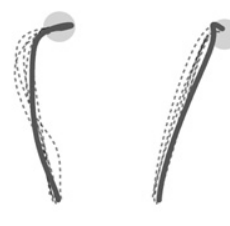

Left Center Right

Fig. 2. Top view of the hand paths of the patient (dark line) and all control participants (dashed lines). (A) Last movement toward the central target in the normal force field (pre-test, no platform rotation). (B) First and last trials in the novel force field (adaptation to the platform rotation). (C) First trial in the normal force field (post-test, no rotation)

paths when analysing the standard deviation of initial movement direction (Fig. 1D) which was greater than that of controls $(p<0.01)$. In summary, baseline testing clearly showed that GL's motor performance was impaired with respect to age-matched, healthy participants.

\subsection{Reaching movement in a novel force field}

Fig. 2 shows that with respect to the last reaching movement toward the central target in PRE-rotation test, the first movement performed in the PER-rotation phase was substantially altered for

all participants, including the deafferented patient. The first movement in the new force field was performed toward the central target by all participants. None of the participants had ever been tested on a rotating platform like the one used in the present study. As the perturbation elicited by the counterclockwise rotation was new to them, Coriolis forces deviated the arm movements clockwise, i.e., rightward.

The adaptation process that took place afterward is illustrated by the difference between the first and last movements in the modified force field: by being exposed to the platform rotation, participants restored the movement performance observed during the baseline, PRE-rotation test. This was observed for the control participants but also for the deafferented patient: therefore, all participants were able to reach toward the target by producing straight movements, indicating that the nervous system could take into account the novel force field to update motor commands. This was further evidenced by the after-effect observed once the platform had stopped rotating: reaching movements of both the controls and the deafferented patient were initially misdirected leftward. These classic after-effects are usually thought to reflect the updating of the internal representation of limb dynamics (Lackner and DiZio, 1994; Shadmehr \& Mussa-Ivaldi, 1994).

Statistical analysis showed that the consequences of the novel force field on arm trajectory were similar for the patient and the controls (Fig. 2B). The perturbation was substantial since for the first movement in the new force field, controls' initial movement direction was shifted leftward by $12.8^{\circ}$ on average with respect to baseline performance assessed in the PRE-test. In comparison, the first movement of the patient in the new force field was shifted by $9.6^{\circ}$, a value not statistically different from that of controls $(p>0.21)$. Because Coriolis forces depend on arm movement velocity, it should be noted that the peak velocity of the first reaching movement of the patient was not significantly different from that of controls ( 1.6 and $1.7 \pm 0.1 \mathrm{~m} / \mathrm{s}$, respectively; $p>0.13$ ).

It can be seen in Fig. 2B that for the first reach in the new dynamic environment, movement trajectory was initially misdirected before reversing back toward the target. In fact, absolute endpoint errors were not significantly influenced by any of the experimental factors $(p>0.17)$. When analyzing the lateral errors at movement offset, we found that only the interaction Test $\times$ Trial was significant $\left(F_{10,50}=2.8 ; p<0.01\right)$ but post-hoc analysis did not show any significant differences between PRE-rotation and PER-rotation trials. Therefore, the rotation did not significantly affect endpoint positioning, which likely reflected planning and online corrective processes. The time of maximum perpendicular deviation was used to assess the time of online correction of the trajectory in the first trial of the PER-rotation condition. This analysis indicated that on average, controls' movements were corrected after $351 \pm 28 \mathrm{~ms}$ while the patient's movement was
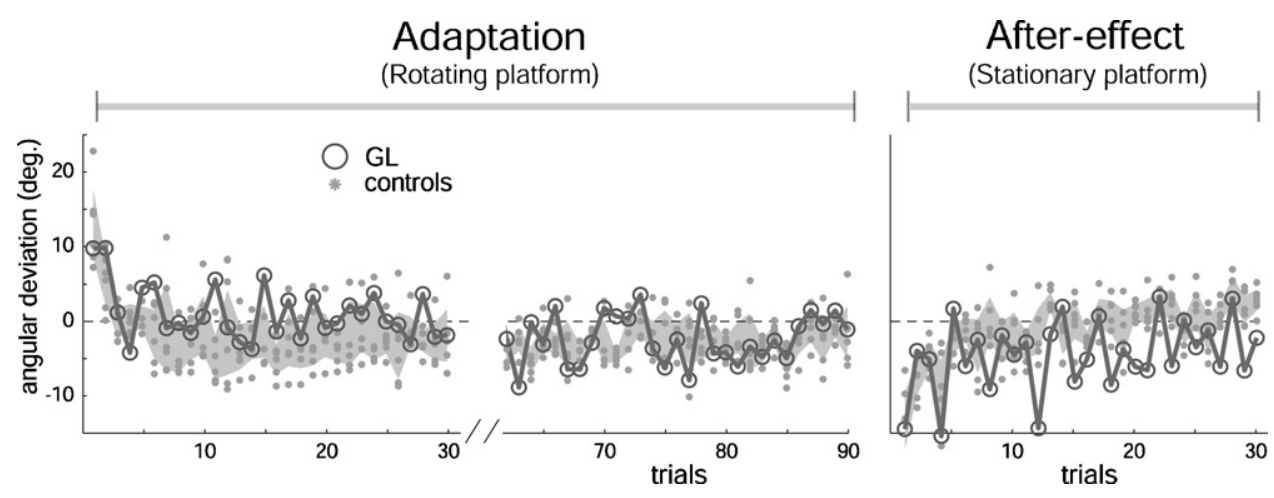

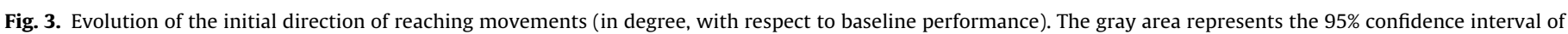
control participants. 
corrected after $372 \mathrm{~ms}$. This $21 \mathrm{~ms}$ difference was not significant $(p>0.12)$.

To assess the adaptive modification of planning processes, we mainly analyzed initial movement direction. Fig. 3 shows that control participants rapidly adapted their motor planning to the new forces acting on the moving limb but also that the patient, deprived of proprioception, was able to adapt to the new force field. Indeed, it can be seen that over the 90 movements performed toward three different targets in the new force field, errors in initial movement direction were large for the first movement but rapidly decreased. The ANOVA on controls' initial movement direction revealed a significant interaction Test $\times$ Trial $\left(F_{10,50}=20.2 ; p<0.001\right)$. Post-hoc analysis showed that the first movement in the novel force field, directed toward the central target for all participants, was significantly deviated rightward with respect to all baseline movements $(p<0.001)$. The second movement, which was toward the left target for all participants, was also significantly deviated rightward $(p<0.05)$. On the third trial, always requiring a movement toward the right target, the effect of the force field did not significantly influence anymore controls' initial direction $(p>0.61)$. Thus, it took three trials for controls to restore the initial direction of their movements to baseline level.

It can be seen in Fig. 3 that it also took three trials for the deafferented patient to restore baseline performance in terms of initial movement direction. In fact, for the first, second and third movements in the modified force field, we found that the initial direction of the patient's movement did not significantly differ from that of controls ( $p>0.21,0.09$ and 0.23 , respectively). Inspecting the peak velocities of GL's first three movements $(1.6,1.8$ and $2.3 \mathrm{~m} / \mathrm{s}$ ) in the novel force field revealed that the patient did not strategically slow down her movements to improve accuracy. Thus, when assessing the time course of adaptation, we observed that the deafferented patient adapted to novel limb dynamics as rapidly as control participants. These results support the idea that all participants, including the deafferented patient, could use error signals from a given trial to update, in the next trial, the motor commands according to the novel force and that such learning generalized to the neighboring target directions (Gandolfo, Mussa-Ivaldi, \& Bizzi, 1996; Mattar \& Ostry, 2007). It should be noted that for the forth, fifth and sixth trials, the patient's initial direction differed from controls $(p<0.01$, 0.05 and 0.05 , respectively), a finding suggesting that force adaptation could be slightly slowed down in deafferented patients.

\subsection{After-effects in a normal force field after adaptation to a modified force field}

Figs. 2C and 3 show that all control participants exhibited an after-effect on the first trial of the POST-test. Once the rotation stopped, i.e., when the normal force field was restored, participants did not reach straight to the central target but initially directed their reaching movements leftward with respect to target direction. This was well reflected by the controls' initial movement direction which shifted by $11.7^{\circ}$ on average, a significant difference with respect to PRE-test trials $(p<0.001)$. An important result of the present study was that the patient's initial movement direction $\left(-12.7^{\circ}\right)$ was also substantially shifted leftward. Moreover, it did not significantly differ from that of controls $(p>0.59)$. On average, time to maximum perpendicular deviation was $347 \pm 16 \mathrm{~ms}$ for control participants while it was $362 \mathrm{~ms}$ for the patient, the difference just failing to be significant $(p>0.06)$. This suggests that movement trajectory tended to be corrected faster for controls than for the patient. Overall, the first reaching movement of the patient after the removal of the novel force field was similar to that of controls.

Fig. 3 shows that de-adaptation, or re-adaptation to the normal force field, was rapid for all participants, including the deafferented patient. For controls, the second movement, which was toward the right target for all participants, was still significantly deviated leftward with respect to pre-test trials $(p<0.05)$. On the third trial however, there were no significant differences in initial movement direction between post- and pre-test trials $(p>0.15)$. Thus, controls restored baseline initial direction in three post-test trials. The initial direction of the patient's second movement slightly differed from that of controls $(p<0.05)$ but in the third trial, it was not significantly different from that of controls $(p>0.69)$. We conclude that after-effects and de-adaptation were similar for the patient and control participants. However, we noticed that the patient could not restore baseline performance for movements toward the left target: since those movements required the most shoulder motion with respect to movements to other targets, this finding is consistent with the idea that proprioceptive deafferentation results in deficits in interjoint coordination (Sainburg et al., 1995; Messier et al., 2003).

\section{Discussion}

In the present study, we asked whether proprioception is necessary to update the internal representation of limb dynamics. We tested the motor behavior of a deafferented patient, totally deprived of proprioception below the nose, on a rotating platform which produced a novel force field deviating the arm movement from its intended trajectory. We provided participants with full visual feedback of hand position as it conveys critical information when one has to reach toward visual targets (Sarlegna \& Sainburg, 2007): participants had thus the opportunity to use visual error signals to adapt motor commands to the altered environment (Sarlegna, Gauthier, \& Blouin, 2007). Results demonstrated that the proprioceptively deafferented patient was able to adapt to the new force field as well as control participants. Moreover, the patient showed after-effects when tested at rest after the rotation, indicating that it is possible to update the central representation of limb dynamics without proprioception. Therefore, our findings indicate that dynamic adaptation, i.e., the adaptation of central control signals to the force environment, can develop on the sole basis of visual feedback.

The fact that adaptation could be observed without proprioception is consistent with previous work showing that proprioception is not absolutely necessary to adapt to visual perturbations like those produced with prisms (Bard, Fleury, Teasdale, Paillard, \& Nougier, 1995; Taub \& Goldberg, 1974) or virtual-reality set-ups (Bernier, Chua, Bard, \& Franks, 2006; Fourneret, Paillard, Lamarre, Cole, \& Jeannerod, 2002; Ingram et al., 2000). Bernier et al. (2006) used a visuo-motor rotation paradigm such that when participants initiated a reaching movement, the visual representation of the hand was rotated by $30^{\circ}$. In their study, GL (the deafferented patient also tested in the present study) was able to adapt as efficiently as control participants. The findings of Bernier et al. (2006) thus support the idea that proprioceptively deafferented patients can process visually detected errors in movement trajectory in order to adaptively modify visuo-motor transformations. It has been suggested that such visually based adaptation rely on the updating of the spatio-temporal coordinates of the desired movement or, in other words, of the kinematic plan (Krakauer et al., 1999; Wang \& Sainburg, 2005). Even though visual and force perturbations both result in a discrepancy between the desired movement and the visually perceived movement, adaptation to a new force field would be different as it is thought to rely on the updating of the central representation of limb dynamics, the kinematic plan remaining invariant (Lackner \& DiZio, 2005; Shadmehr \& Mussa-Ivaldi, 1994). In the present study, the proprioceptively deafferented patient could only use visual feedback to update the representation of limb 
dynamics. In fact, she did report seeing unexpected deviations in movement trajectory on the first trials of platform rotation. Our results show that a deafferented patient can use visual error signals to adapt to a substantial modification of the force environment, extending previous knowledge on the adaptive capacity of patients deprived of proprioception.

It has been suggested that kinematic adaptation, assessed with visuo-motor perturbations, would mainly rely on vision while dynamic adaptation, assessed with mechanical perturbations, would mainly rely on proprioception (Krakauer et al., 1999; Pipereit et al., 2006). However, previous studies already provided some evidence that proprioceptively deafferented patients can use vision to obtain dynamic information (Cole \& Sedgwick, 1992; Fleury et al., 1995). For instance, Fleury et al. (1995) observed that when patient GL was asked to judge the weight of different handheld objects, her strategy was to produce the same set of motor commands and visually analyze her hand displacements. When movement velocity was high, the transported object was judged to be light and when movement velocity was low, the object was judged to be heavy. This clearly illustrates the idea that vision can be a useful source of information to infer dynamic properties. This is consistent with a study by Ghez et al. (1995) which showed improvements in motor performance of proprioceptively deafferented patients when visual information of limb position was available concurrently or prior to movement. Ghez et al. (1995) suggested that a visual updating of the internal model of limb dynamics is efficient when proprioception cannot be used to adapt motor commands to the biomechanical characteristics of the limb. Further experiments are necessary to determine whether visual information of starting position, or visual knowledge of results, is sufficient for deafferented subjects to adapt to a new force field as in the present study. However, it should be noted that even with full vision, deafferented patients cannot control multi-joint movements as efficiently as healthy individuals (Sainburg et al., 1993). This was also shown in the present study since, in baseline testing prior to any perturbation, motor deficits of the deafferented patient were evident with respect to control participants.

Baseline data revealed that control participants performed better with visual and proprioceptive information than the proprioceptively deafferented patient who could only use visual information, a finding supporting the idea that multi-sensory integration enhances motor control. However, the deafferented patient adapted as efficiently as controls to the perturbation. Thus, we did not observe advantages in multimodal conditions (vision and proprioception for controls) with respect to the unimodal condition (only vision for the deafferented patient) to learn the novel force field. This is consistent with the studies by Franklin et al. (2007) and Scheidt et al. (2005) which reported that a visual and proprioceptive condition did not substantially differ from a proprioceptive condition in classic dynamic adaptation protocols. More complex force fields, and more target locations in the workspace, could be employed to further challenge motor adaptation abilities and determine more precisely how multi-sensory integration contributes to motor adaptation in new dynamic environments. In fact, previous studies showed that participants can adapt to a new force field on the sole basis of proprioceptive feedback (DiZio \& Lackner, 2000; Lackner \& DiZio, 1994). This highlights the importance of proprioceptive signals for adapting to a new force field, an idea further supported by Pipereit et al. (2006) who showed that degraded proprioception (by means of vibration) impairs adaptation to novel limb dynamics. This body of evidence indicates that in healthy participants, proprioceptive signals are important for the adaptive learning of a novel force field.

While our suggestion that vision contributes to the updating of the central representation of limb dynamics stems from behavioral findings on a proprioceptively deafferented patient, it is consis- tent with previous studies on healthy humans. Indeed, Bourdin et al. $(2001,2006)$ showed that visual information of the limb, even if only available prior to the reaching movement, enhances adaptation to a complex, multi-force environment with respect to conditions without visual information. Scheidt et al. (2005) further studied the role of vision in the adaptation to dynamic perturbations by using a virtual reality set-up combined with a robotic manipulandum. The virtual environment was used to eliminate any visually detected errors so that when the arm movement was actually deviated by the robot, participants saw their hand going straight to the target. Even though hand path deviations could be felt, no adaptation was observed to the new force field. This study thus showed that visual feedback is important in dynamic adaptation by demonstrating that as long as movement control looks efficient, no adaptation is necessary.

Mattar and Gribble (2005) employed a different protocol to explore the role of vision in adapting motor commands to a novel dynamic environment: they asked participants to watch another participant learning to reach for targets in a novel force field created by a robotic device. After the observation period, participants performed better when later tested in the same force field than subjects who did not previously observe the learning phase. The authors suggested that participants could use visual signals to create an internal representation of the force field. In the present study, by testing a patient with a severe sensory neuropathy, we provide new findings supporting and extending the idea that vision is useful for adapting the motor behavior to the physical constraints acting on the body.

It has been shown that force-field adaptation critically depends on the cerebellum (Maschke, Gomez, Ebner, \& Konczak, 2004; Smith \& Shadmehr, 2005). Since the cerebellum is thought to act as a comparator of predicted and perceived state of the limb (Shadmehr \& Wise, 2005; Wolpert, Miall, \& Kawato, 1998), adapting to new limb dynamics might rely on the updating of cerebellar networks devoted to active and passive limb motion (Shadmehr \& Holcomb, 1997; Thickbroom, Byrnes, \& Mastaglia, 2003). We speculate that visual feedback of hand motion may contribute, in addition to proprioceptive feedback, to the updating of the motor representations stored in the cerebellum, in order to restore adequate motor performance in the modified force field. This suggestion is consistent with the idea that the cerebellum is involved in the integration of visual and proprioceptive signals related to hand position (Hagura et al., 2009).

We investigated the behavior of a deafferented patient to study the role of visual and proprioceptive signals in motor learning and it should be recalled that patient GL has been deafferented for almost 30 years at the time of the study. She could thus rely on cognitive strategies to optimize her motor behavior (Fleury et al., 1995). However, numerous behavioral studies showed that healthy humans also rely on cognitive resources when learning to reach in a new environment (Ingram et al., 2000; Malfait \& Ostry, 2004; Taylor \& Thoroughman, 2008). Moreover, neurophysiological studies showed that during the early phase of motor adaptation, there is an increased activity in frontal brain areas associated with cognitive functions (Della-Maggiore \& McIntosh, 2005; Krakauer et al., 2004; Shadmehr \& Holcomb, 1997). In the present study, both the patient and controls likely used cognitive resources to adapt to the new force field but we did not observe significant differences in the time course of adaptation between the patient and controls. Moreover, all participants exhibited similar after-effects, i.e., misdirected reaches, despite being informed that the experimental platform stopped rotating. Thus, in contrast to studies using robotic devices, the procedure used in our study allowed participants to easily detect when the novel force field was removed since this precisely corresponded to the end of the platform deceleration. One would expect that if the patient relied more on cognitive strategies 
than controls, she would have exhibited a reduced after-effect with respect to controls as she could have moved the hand straighter toward the target by selecting motor commands more adapted to the current, normal context. In fact, the similar after-effects for the proprioceptively deafferented patient and control participants suggest that cognitive strategies contributed to a similar extent to motor learning for the patient and the controls.

The ability of the patient to adapt to the new force field may have been influenced by a cerebral reorganization in response to her severe large-fiber neuropathy. It is well known that after proprioceptive deafferentation, there is a reorganization of the primary somatosensory cortex (S1), even in the mature human brain. Pons et al. (1991) showed that after surgical limb deafferentation of nonhuman primates, the $S 1$ area that previously responded to tactile stimulation of the limb now responded to the tactile stimulation of the face. It is unknown whether such cortical reorganization affected GL. However, it has been shown that after proprioceptive deafferentation, S1 takes up some motor processing functions or some processing of other sensory inputs such as auditory inputs and visual inputs (Weeks, Gerloff, Dalakas, \& Hallett, 1999) and possibly vestibular inputs (Blouin, Teasdale, \& Mouchnino, 2007; Day \& Cole, 2002). Cross-modal plasticity, which has been well characterized in impaired individuals (for reviews, Bavelier, Dye, \& Hauser, 2006; Pascual-Leone, Amedi, Fregni, \& Merabet, 2005), could explain the ability of the proprioceptively deafferented patient to use vision for adapting her central control signals to a novel dynamic environment. However, Balslev, Miall, and Cole (2007) suggested $a$ contrario that proprioceptive deafferentation, induced in healthy participants by repetitive transcranial magnetic stimulation over $\mathrm{S} 1$, slows down the online processing of visual feedback of the moving hand. It thus remains unclear whether cross-modal plasticity confers visual advantages, or others, to proprioceptively deafferented patients.

\section{Acknowledgments}

We would like to thank GL for being such a great patient, as well as Annie Schmied for organizing GL venue. We are grateful to Gabriel M. Gauthier for theoretical as well as technical inputs. Thanks also to Alain Donneaud for his assistance when building the set-up, to Frank Buloup for his programming expertise and Docometre software development, and to Naomi Russo for her continuous support. This work received financial support from the DGA, CNRS and the University of the Mediterranean.

\section{References}

Balslev, D., Miall, R. C., \& Cole, J. (2007). Proprioceptive deafferentation slows down the processing of visual hand feedback. Journal of Vision, 7, 1-7.

Bard, C., Fleury, M., Teasdale, N., Paillard, J., \& Nougier, V. (1995). Contribution of proprioception for calibrating and updating the motor space. Canadian Journal of Physiology \& Pharmacology, 73, 246-254.

Bavelier, D., Dye, M. W., \& Hauser, P. C. (2006). Do deaf individuals see better? Trends in Cognitive Sciences, 10, 512-518.

Bernier, P. M., Chua, R., Bard, C., \& Franks, I. M. (2006). Updating of an internal model without proprioception: A deafferentation study. Neuroreport, 17, 1421-1425.

Blouin, J., Bard, C., Teasdale, N., Paillard, J., Fleury, M., Forget, R., et al. (1993). Reference systems for coding spatial information in normal subjects and a deafferented patient. Experimental Brain Research, 93, 324-331.

Blouin, J., Teasdale, N., \& Mouchnino, L. (2007). Vestibular signal processing in a subject with somatosensory deafferentation: The case of sitting posture. BMC Neurology, 29, 7-25.

Bourdin, C., Gauthier, G. M., Blouin, J., \& Vercher, J.-L. (2001). Visual feedback of the moving arm allows complete adaptation of pointing movements to centrifugal and Coriolis forces. Neuroscience Letters, 301, 25-28.

Bourdin, C., Bringoux, L., Gauthier, G. M., \& Vercher, J.-L. (2006). Vision of the hand prior to movement onset allows full motor adaptation to a multi-force environment. Brain Research Bulletin, 71, 101-110.

Brown, L. E., Rosenbaum, D. A., \& Sainburg, R. L. (2003). Limb position drift: Implications for control of posture and movement. Journal of Neurophysiology, 90, 3105-3118.
Buneo, C. A., Boline, J., Soechting, J. F., \& Poppele, R. E. (1995). On the form of the internal model for reaching. Experimental Brain Research, 104, 467479.

Coello, Y., Orliaguet, J. P., \& Prablanc, C. (1996). Pointing movement in an artificial perturbing inertial field: A prospective paradigm for motor control study. Neuropsychologia, 34, 879-892.

Cole, J. D., \& Sedgwick, E. M. (1992). The perceptions of force and of movement in a man without large myelinated sensory afferents below the neck. Journal of Physiology, 449, 503-515.

Cooke, J. D., Brown, S., Forget, R., \& Lamarre, Y. (1985). Initial agonist burst duration changes with movement amplitude in a deafferented patient. Experimental Brain Research, 60, 184-187.

Cordo, P., Gurfinkel, V. S., Bevan, L., \& Kerr, G. K. (1995). Proprioceptive consequences of tendon vibration during movement. Journal of Neurophysiology, 74, $1675-1688$.

Day, B. L., \& Cole, J. (2002). Vestibular-evoked postural responses in the absence of somatosensory information. Brain, 125, 2081-2088.

Della-Maggiore, V., \& McIntosh, A. R. (2005). Time course of changes in brain activity and functional connectivity associated with long-term adaptation to a rotational transformation. Journal of Neurophysiology, 93(4), 2254-2262.

DiZio, P., \& Lackner, J. R. (2000). Congenitally blind individuals rapidly adapt to Coriolis force perturbations of their reaching movements. Journal of Neurophysiology, $84,2175-2180$

Fleury, M., Bard, C., Teasdale, N., Paillard, J., Cole, J., Lajoie, Y., et al. (1995). Weight judgment. The discrimination capacity of a deafferented subject. Brain, 118 1149-1156.

Fourneret, P., Paillard, J., Lamarre, Y., Cole, J., \& Jeannerod, M. (2002). Lack of conscious recognition of one's own actions in a haptically deafferented patient. Neuroreport, 25, 541-547.

Franklin, D. W., So, U., Burdet, E., \& Kawato, M. (2007). Visual feedback is not necessary for the learning of novel dynamics. Public Library of Science ONE, 19 , e1336.

Gandolfo, F., Mussa-Ivaldi, F. A., \& Bizzi, E. (1996). Motor learning by field approximation. Proceedings of the National Academy of Sciences United States of America 30, 3843-3846.

Gauthier, G. M., \& Mussa Ivaldi, F. (1988). Oculo-manual tracking of visual targets in monkey: Role of the arm afferent information in the control of arm and eye movements. Experimental Brain Research, 73, 138-154.

Ghez, C., Gordon, J., \& Ghilardi, M. F. (1995). Impairments of reaching movements in patients without proprioception. II. Effects of visual information on accuracy. Journal of Neurophysiology, 73, 361-372.

Hagura, N., Oouchida, Y., Aramaki, Y., Okada, T., Matsumura, M., Sadato, N., et al. (2009). Visuokinesthetic perception of hand movement is mediated by cerebrocerebellar interaction between the left cerebellum and right parietal cortex. Cerebral Cortex, 19, 176-186.

Ingram, H. A., van Donkelaar, P., Cole, J., Vercher, J. L., Gauthier, G. M., \& Miall, R. C. (2000). The role of proprioception and attention in a visuomotor adaptation task. Experimental Brain Research, 132, 114-126.

Krakauer, J. W., Ghilardi, M. F., \& Ghez, C. (1999). Independent learning of internal models for kinematic and dynamic control of reaching. Nature Neuroscience, 2 1026-1031.

Krakauer, J. W., Ghilardi, M. F., Mentis, M., Barnes, A., Veytsman, M., Eidelberg, D., et al. (2004). Differential cortical and subcortical activations in learning rotations and gains for reaching: A PET study. Journal of Neurophysiology, 91, 924-933.

Lackner, J. R., \& DiZio, P. (1994). Rapid adaptation to Coriolis force perturbations of arm trajectory. Journal of Neurophysiology, 72, 299-313.

Lackner, J. R., \& DiZio, P. (2005). Motor control and learning in altered dynamic environments. Current Opinion in Neurobiology, 15, 653-659.

Malfait, N., \& Ostry, D. J. (2004). Is interlimb transfer of force-field adaptation a cognitive response to the sudden introduction of load? Journal of Neuroscience 24, 8084-8089.

Maschke, M., Gomez, C. M., Ebner, T. J., \& Konczak, J. (2004). Hereditary cerebellar ataxia progressively impairs force adaptation during goal-directed arm movements. Journal of Neurophysiology, 91, 230-238.

Mattar, A. A., \& Gribble, P. L. (2005). Motor learning by observing. Neuron, 7, 153-160

Mattar, A. A., \& Ostry, D. J. (2007). Modifiability of generalization in dynamics learning. Journal of Neurophysiology, 98, 3321-3329.

Messier, J., Adamovich, S., Berkinblit, M., Tunik, E., \& Poizner, H. (2003). Influence of movement speed on accuracy and coordination of reaching movements to memorized targets in three-dimensional space in a deafferented subject. Experimental Brain Research, 150, 399-416.

Mott, F. W., \& Sherrington, C. S. (1895). Experiments upon the influence of sensory nerves upon movement and nutrition of the limbs. Proceedings of the Royal Society, 57, 481-488.

Nougier, V., Bard, C., Fleury, M., Teasdale, N., Cole, J., Forget, R., et al. (1996). Control of single-joint movements in deafferented patients: Evidence for amplitude coding rather than position control. Experimental Brain Research, 109, 473-482.

Olausson, H., Lamarre, Y., Backlund, H., Morin, C., Wallin, B. G., Starck, G., et al. (2002). Unmyelinated tactile afferents signal touch and project to insular cortex. Nature Neuroscience, 9, 900-904.

Pascual-Leone, A., Amedi, A., Fregni, F., \& Merabet, L. B. (2005). The plastic human brain cortex. Annual Reviews of Neuroscience, 28, 377-401.

Pipereit, K., Bock, O., \& Vercher, J. L. (2006). The contribution of proprioceptive feedback to sensorimotor adaptation. Experimental Brain Research, 174, 45-52.

Polit, A., \& Bizzi, E. (1979). Characteristics of motor programs underlying arm movements in monkeys. Journal of Neurophysiology, 42, 183-194. 
Pons, T. P., Garraghty, P. E., Ommaya, A. K., Kaas, J. H., Taub, E., \& Mishkin, M. (1991) Massive cortical reorganization after sensory deafferentation in adult macaques. Science, 252, 1857-1860.

Rossit, S., \& Harvey, M. (2008). Age-related differences in corrected and inhibited pointing movements. Experimental Brain Research, 185, 1-10.

Rothwell, J. C., Traub, M. M., Day, B. L., Obeso, J. A., Thomas, P. K., \& Marsden, C. D. (1982). Manual motor performance in a deafferented man. Brain, 105, 515-542.

Sainburg, R. L., Poizner, H., \& Ghez, C. (1993). Loss of proprioception produces deficits in interjoint coordination. Journal of Neurophysiology, 70, 2136-2147.

Sainburg, R. L., Ghilardi, M. F., Poizner, H., \& Ghez, C. (1995). Control of limb dynamics in normal subjects and patients without proprioception. Journal of Neurophysiology, 73, 820-835.

Sainburg, R. L., Lateiner, J. E., Latash, M. L., \& Bagesteiro, L. B. (2003). Effects of altering initial position on movement direction and extent. Journal of Neurophysiology, 89, 401-415.

Sanes, J. N., Mauritz, K. H., Dalakas, M. C., \& Evarts, E. V. (1985). Motor control in humans with large-fiber sensory neuropathy. Human Neurobiology, 4, 101114.

Sarlegna, F. R. (2006). Impairment of online control of reaching movements with aging: A double-step study. Neuroscience Letters, 403, 309-314.

Sarlegna, F. R., \& Sainburg, R. L. (2007). The effect of target modality on visual and proprioceptive contributions to the control of movement distance. Experimental Brain Research, 176, 267-280.

Sarlegna, F. R., \& Sainburg, R. L. (2009). The roles of vision and proprioception in planning and controlling movements. In D. Sternad (Ed.), Progress in motor control. A multidisciplinary perspective (pp. 315-333). New York: Springer.

Sarlegna, F. R., Gauthier, G. M., Bourdin, C., Vercher, J.-L., \& Blouin, J. (2006) Internally-driven control of reaching movement: A study on a proprioceptivelydeafferented subject. Brain Research Bulletin, 69, 404-415.

Sarlegna, F. R., Gauthier, G. M., \& Blouin, J. (2007). Sensori-motor adaptation: Contribution of visual, proprioceptive and verbal cues. Journal of Motor Behavior, 39, 247-258.

Scheidt, R. A., Conditt, M. A., Secco, E. L., \& Mussa-Ivaldi, F. A. (2005). Interaction of visual and proprioceptive feedback during adaptation of human reaching movements. Journal of Neurophysiology, 93, 3200-3213.
Shadmehr, R., \& Holcomb, H. H. (1997). Neural correlates of motor memory consolidation. Science, 27, 821-825.

Shadmehr, R., \& Mussa-Ivaldi, F. A. (1994). Adaptive representation of dynamics during learning of a motor task. Journal of Neuroscience, 14, 3208-3224

Shadmehr, R., \& Wise, S. P. (2005). The computational neurobiology of reaching and pointing. In R. Shadmehr, \& S. P. Wise (Eds.), A Foundation for Motor Learning. Cambridge, MA: MIT Press.

Smith, M. A., \& Shadmehr, R. (2005). Intact ability to learn internal models of arm dynamics in Huntington's disease but not cerebellar degeneration. Journal of Neurophysiology, 93, 2809-2821.

Sober, S. J., \& Sabes, P. N. (2005). Flexible strategies for sensory integration during motor planning. Nature Neuroscience, 8, 490-497.

Taub, E., \& Goldberg, I. A. (1974). Use of sensory recombination and somatosensory deaffrentation techniques in the investigation of sensory-motor integration. Perception, 3, 393-408

Taylor, J. A., \& Thoroughman, K. A. (2008). Motor adaptation scaled by the difficulty of a secondary cognitive task. Public Library of Science ONE, 18, e2485.

Thickbroom, G. W., Byrnes, M. L., \& Mastaglia, F. L. (2003). Dual representation of the hand in the cerebellum: activation with voluntary and passive finger movement. Neuroimage., 3, 670-674

Tong, C., Wolpert, D. M., \& Flanagan, J. R. (2002). Kinematics and dynamics are not represented independently in motor working memory: Evidence from an interference study. Journal of Neuroscience, 22, 1108-1113.

Vercher, J.-L., Gauthier, G. M., Guédon, O., Blouin, J., Cole, J., \& Lamarre, Y. (1996). Self-moved target tracking in control and deafferented subjects: Role of arm motor command efferent copy and proprioception in arm-eye coordination. Journal of Neurophysiology, 76, 11331144.

Wang, J., \& Sainburg, R. L. (2005). Adaptation to visuomotor rotations remaps movement vectors, not final positions. Journal of Neuroscience, 25, 4024-4030.

Weeks, R. A., Gerloff, C., Dalakas, M., \& Hallett, M. (1999). PET study of visually and non-visually guided finger movements in patients with severe pan-sensory neuropathies and healthy controls. Experimental Brain Research, 128, 291-302.

Wolpert, D. M., Miall, R. C., \& Kawato, M. (1998). Internal models in the cerebellum. Trends in Cognitive Sciences, 2, 338-347. 